\title{
Open
}

\section{The natural history of MPS I: global perspectives from the MPS I Registry}

\author{
Michael Beck, MD1, Pamela Arn, MD², Roberto Giugliani, MD, PhD, MSc$c^{3}$, Joseph Muenzer, MD, PhD', \\ Torayuki Okuyama, $\mathrm{MD} \mathrm{PhD}^{5}$, John Taylor, $\mathrm{MS}^{6}$ and Shari Fallet, $\mathrm{DO}^{6}$
}

Purpose: In this study, we aimed to describe the natural history of mucopolysaccharidosis I.

Methods: Data from 1,046 patients who enrolled in the MPS I Registry as of August 2013 were available for descriptive analysis. Only data from untreated patients and data prior to treatment for patients who received treatment were considered. Age at symptom onset, diagnosis, and treatment initiation were examined by geographic region and phenotype (from most to least severe: Hurler, HurlerScheie, and Scheie). For each symptom, frequency and age at onset were examined.

Results: Natural history data were available for 987 patients. Most patients were from Europe $(45.5 \%)$, followed by North America (34.8\%), Latin America (17.3\%), and Asia Pacific (2.4\%). Phenotype distribution was $60.9 \%$ for Hurler, $23.0 \%$ for Hurler-Scheie, and
$12.9 \%$ for Scheie (3.2\% undetermined) syndromes. Median age at symptom onset for Hurler, Hurler-Scheie, and Scheie syndromes was 6 months, 1.5 years, and 5.3 years, respectively; median age at treatment initiation was 1.5 years, 8.0 years, and 16.9 years, respectively. Coarse facial features and corneal clouding were among the most common symptoms in all three phenotypes.

Conclusion: A delay between symptom onset and treatment exists, especially in patients with attenuated mucopolysaccharidosis I. A better understanding of disease manifestations may help facilitate prompt diagnosis and treatment and improve patient outcomes.

Genet Med advance online publication 27 March 2014

Key Words: MPS I; natural history; registry; symptoms

\section{INTRODUCTION}

Mucopolysaccharidosis type I (MPS I) is a rare autosomal recessive disease caused by a deficiency of $\alpha$-L-iduronidase, an enzyme required for the degradation of the glycosaminoglycans dermatan and heparan sulfate. The estimated incidence of MPS I is 1 in every 100,000 live births. Due to the chronic and progressive accumulation of glycosaminoglycans in the lysosomes of cells throughout the body, patients affected by this devastating disease experience multiorgan dysfunction leading to considerable morbidity in most patients, and early mortality in those most severely affected. ${ }^{1}$

Like most other metabolic inherited diseases, MPS I displays significant variability in its presentation and course. This may be due to differences among patients in the severity of the underlying mutation(s) and consequent degree of residual enzyme activity; however, other factors may also contribute to the well-known phenotypic heterogeneity. ${ }^{2}$ Historically, MPS I has been classified into three syndromesHurler, Hurler-Scheie, and Scheie-though it is now widely accepted that overlap in symptomatology exists among these subtypes. ${ }^{1}$ Hurler syndrome, the most severe form of MPS I, typically involves significant developmental delay and cognitive decline, along with characteristic coarse facial features, joint stiffness and contractures, short stature, and respiratory, cardiac, and hepatic disease. Symptoms emerge shortly after birth and progress rapidly, such that most patients with Hurler syndrome die within the first decade of life. At the other end of the MPS I disease spectrum, Scheie syndrome involves later onset of typically milder symptoms and a slower disease progression. While patients with the Scheie phenotype usually develop significant disease-related morbidity, they show normal intelligence and survive into adulthood. Hurler-Scheie syndrome represents an intermediate phenotype that is characterized by mild or no cognitive impairment but includes somatic symptoms that reduce life expectancy into the second or third decade of life. Delineation of the different MPS I phenotypes can be challenging and is largely driven by consideration of the age of symptom onset and rate of disease progression as well as a patient's genotype. ${ }^{3}$

Historically, treatment of MPS I was restricted to palliative care and symptom-based interventions, including surgery (e.g., adenotonsillectomy, hernia repair, ventriculoperitoneal shunt, cardiac valve replacement, carpal tunnel release, and spinal decompression); physical, occupational, and speech therapies; respiratory support; hearing aids; and medications for pain and gastrointestinal disturbances. Since 1981, hematopoietic stem cell transplantation (HSCT) has been used to treat MPS I. When successful, it is a one-time procedure that can prolong survival, preserve cognitive function, and reduce some somatic features of the disease ${ }^{4,5}$ However, due to its significant 
morbidity, HSCT is reserved for patients with the most severe form of MPS I, Hurler syndrome. HSCT is typically recommended for patients under 2 years of age with normal cognition (intelligence quotient $>70$ ) because early intervention increases the likelihood of maintaining cognitive abilities. For patients with Hurler-Scheie and Scheie syndromes, enzyme replacement therapy (ERT) with laronidase (recombinant human $\alpha$-L-iduronidase; Aldurazyme) is the primary treatment option. ${ }^{6-10}$ Laronidase is administered weekly via intravenous infusion and is a lifelong therapy.

The success of both HSCT and ERT may depend on early initiation of treatment. Developmental outcomes are better when transplantation is performed before 24 months of age, ${ }^{11}$ and laronidase may also be more beneficial when started early in life, based on the suggestion from two case reports on siblings with Hurler-Scheie syndrome. ${ }^{12,13}$ Unfortunately, early diagnosis (and hence early initiation of treatment) is limited by several factors, including the rarity of the disease, the wide variability in clinical presentation and disease course, and the nonspecific nature of some of the early manifestations of the disease. As is true of other rare genetic diseases, MPS I awareness among physicians is also hampered by a general lack of familiarity with its symptoms and course.

With the advent of several potentially lifesaving therapies, the need for better disease recognition and early diagnosis is heightened. The MPS I Registry (ClinicalTrials.gov identifier: NCT00144794) is a global, observational disease database established in 2003 by Genzyme/BioMarin and is maintained by Genzyme, a Sanofi company. ${ }^{12}$ The collection of data in the Registry is aimed at facilitating characterization of the course of disease and tracking clinical outcomes in patients with MPS I. In addition, participating health-care professionals are provided access to aggregate data on MPS I and can query the database for specific information to facilitate the care of their patients with MPS I. Analyses of data from the Registry have provided insights about disease burden, genotype-phenotype correlations, frequency and risks of surgical procedures, and changing treatment trends in MPS I patients. ${ }^{13-15}$ The intent of this analysis is to use data from the Registry to describe the natural history of MPS I by phenotype and across regions in an effort to improve the recognition and prompt diagnosis of this potentially fatal, but treatable, disease.

\section{MATERIALS AND METHODS}

The global MPS I Registry was initiated in April 2003 as part of an effort to help health-care professionals involved in the diagnosis and treatment of MPS I better understand the disease and its management and to help create MPS I disease treatment monitoring guidelines. All data provided for this analysis were obtained as of August 2013. Patient participation in the MPS I Registry is voluntary. Each independent site is responsible for obtaining a patient's informed consent for submitting his/her health information to the Registry and for using and disclosing this information in subsequent aggregate analyses, such as journal articles, annual reports, educational materials, and public health reports. Historically, each independent site has been responsible for determining whether site-specific institutional review board or ethics committee review is required for participation in the Registry in accordance with institutional policies and local laws and regulations. Since December 2012, the MPS I Registry protocol has required sites to submit to and receive approval from an institutional review board or ethics committee. All patient data are anonymized and entered by participating sites in accordance with applicable privacy regulations. At enrollment, detailed medical histories and baseline data are collected and physicians are encouraged to follow a recommended schedule of clinical assessments and regularly report the results of their assessments. ${ }^{12}$

This report is based on Registry data as of August 2013, at which time information was available for 1,046 patients. The Registry includes comprehensive information for treated as well as untreated patients; for the treated patients, data are available from the periods prior to, during, and/or subsequent to treatment. Therefore, a definition of the natural history period and the following patient inclusion criteria were used to select data for the analyses presented here: (i) Known ERT status, (ii) For patients receiving ERT, known date of first infusion, (iii) Known HSCT status, and (iv) For patients who had received HSCT, known date of transplant.

From the initial cohort of 1,046 patients, 987 patients met the inclusion criteria. For patients who had never been treated, the natural history period was defined as the time from birth to the last follow-up recorded in the Registry. Among patients who had been treated with ERT, HSCT, or both, the natural history period was defined as the time from birth to first treatment (either ERT or HSCT).

Ages at first symptom, diagnosis, and treatment initiation (where applicable) were evaluated by region and MPS I phenotype. Symptom prevalence and age of onset of symptoms relating to general appearance and neurologic, cardiac, respiratory, gastrointestinal, and musculoskeletal status were evaluated by phenotype. Only the symptoms observed during the predefined natural history period were considered. Given that not all patients had data reported for every symptom during the natural history period, the assumption was made that symptoms not explicitly reported were not present, and frequencies were calculated as the number of patients with the symptom present divided by all patients in each region and/or phenotype. This assumption means that the reported frequencies may underestimate the true frequency for each symptom. Descriptive statistics were computed for age of onset of each symptom (as indicated by the earliest age each symptom was reported) among patients who experienced the symptom. For those patients with the same symptom reported multiple times, the earliest age was used, corresponding to "onset" or "age first reported."

\section{RESULTS}

\section{Global occurrence of MPS I phenotypes}

A total of 987 MPS I patients with evaluable natural history information were enrolled in the Registry as of August 2013 
(Table 1). The largest proportion of patients was from Europe (45.5\%), with the next largest from North America (34.8\%), followed by Latin America (17.3\%) and Asia Pacific (2.4\%).

The overall phenotypic distribution was 601 (60.9\%) for Hurler, 227 (23.0\%) for Hurler-Scheie, and 127 (12.9\%) for Scheie. Another 32 (3.2\%) patients met the criteria for inclusion in this analysis, but their phenotypes were not reported in the Registry and were therefore considered undetermined or missing. Of note, North America (71.4\%) and Europe (61.5\%) had higher proportions of patients with the severe Hurler phenotype than Latin America (42.7\%) or the Asia Pacific region (29.2\%).

\section{Chronology of symptom onset, diagnosis, and treatment by region}

Median ages at symptom onset, MPS I diagnosis, and first treatment (ERT or HSCT, if applicable) are presented by region and phenotype in Figure 1. Across all regions, patients with Hurler syndrome, the most severe phenotype, were diagnosed and treated earliest, as expected. The median age at symptom onset for these patients was 6 months, and diagnosis and treatment initiation followed quickly thereafter, at median ages of 12 and 18 months, respectively. Patients with Hurler-Scheie and Scheie syndromes, which have the more attenuated presentations, typically experienced initial symptoms sometime after infancy and exhibited a 2- to 4-year gap between onset of symptoms and diagnosis, respectively, and a 4- to 8-year gap between diagnosis and treatment initiation, respectively.

Analyses of age of symptom onset, diagnosis, and treatment initiation based on phenotype and geographic region generally mirrored the results in the overall Registry population, with a few notable exceptions. In North America, treatment initiation for patients with Scheie syndrome was earlier than in the other regions. The median age for treatment initiation for patients diagnosed with Scheie syndrome in North America was 11.7 years, whereas in Europe and Latin America it was 16.9 and 17.7 years, respectively. The Asia Pacific region had an even higher median age of 31.5 years for treatment initiation. The median age of treatment initiation in patients with Hurler-Scheie was also higher in the Asia Pacific region (23.8 years) compared with the overall Registry population (8 years). Another notable finding was the rate of patients with an undetermined or missing phenotype in Latin America (11.1\%), which was much higher compared with the other regions. The median ages of symptom onset, diagnosis, and treatment initiation for patients in Latin America with an undetermined phenotype were 0.7, 1.3 , and 3.3 years, respectively, which is highly comparable to the median ages for patients in Latin America with the Hurler phenotype $(0.7,1.7$, and 3.6 years, respectively), suggesting that some portion of the patients with undetermined phenotypes may, in fact, have Hurler syndrome.

\section{Natural history of MPS I by phenotype}

Symptom frequency by phenotype. Coarse facial features were the most predominant characteristic of all functional and anatomic abnormalities for both the Hurler and the Hurler-Scheie phenotypes, occurring in 86.4 and $72.7 \%$ of these patients, respectively. The symptoms occurring in at least $25 \%$ of patients are summarized by phenotype in Figure 2, and all symptoms, including those occurring in less than $25 \%$ of patients, are summarized by phenotype in Supplementary Table S1 online. Among patients with the Scheie phenotype, $48.0 \%$ presented with coarse facial features. Corneal clouding was noted in all three phenotypes at approximately the same rate: $70.9,68.3$, and $70.1 \%$ for Hurler, Hurler-Scheie, and Scheie, respectively.

Hepatomegaly was present in the majority of patients with Hurler (70.0\%) and Hurler-Scheie $(66.5 \%)$ phenotypes and in approximately half $(48.0 \%)$ of those with the Scheie phenotype. Similarly, splenomegaly was present in $50.9 \%$ of patients with Hurler syndrome and $47.1 \%$ of patients with Hurler-Scheie syndrome but in only $27.6 \%$ of those with the Scheie phenotype. The incidences of hernias were more evenly distributed among the phenotypic groups, with $58.9 \%$ in Hurler, $59.9 \%$ in Hurler-Scheie, and 53.5\% in Scheie patients.

In terms of musculoskeletal abnormalities, kyphosis/gibbus was the only abnormality present in the majority (70.0\%) of patients with Hurler phenotype, and it was reported much less frequently in patients with Hurler-Scheie (33.5\%) and Scheie $(21.3 \%)$ phenotypes. Conversely, joint contractures and carpal tunnel syndrome were present in the majority of Scheie patients (69.3 and $51.2 \%$, respectively) but were less common in Hurler-Scheie patients (57.3 and 27.8\%, respectively) and even less frequent in Hurler patients (37.9 and $7.8 \%$, respectively).

Cardiac valve abnormalities were observed in $67.7 \%$ of Scheie patients, $59.0 \%$ of Hurler-Scheie patients, and $48.9 \%$ of

Table 1 Distribution of MPS I phenotypes by geographic region-all evaluable patients

\begin{tabular}{|c|c|c|c|c|c|}
\hline Geographic region & $\begin{array}{c}\text { Hurler, }^{\mathrm{a}} \\
n(\%)\end{array}$ & $\begin{array}{c}\text { Hurler-Scheie, }^{a} \\
n(\%)\end{array}$ & $\begin{array}{l}\text { Scheie, }^{a} \\
n(\%)\end{array}$ & Undetermined, a $n(\%)$ & $\begin{array}{l}\text { Total, } \\
n(\%)\end{array}$ \\
\hline All regions & $601(60.9)$ & $227(23.0)$ & $127(12.9)$ & $32(3.2)$ & 987 \\
\hline Asia Pacific & $7(29.2)$ & $5(20.8)$ & $12(50.0)$ & 0 & $24(2.4)$ \\
\hline Europe & $276(61.5)$ & $102(22.7)$ & $63(14.0)$ & $8(1.8)$ & $449(45.5)$ \\
\hline Latin America & $73(42.7)$ & $57(33.3)$ & $22(12.9)$ & $19(11.1)$ & $171(17.3)$ \\
\hline North America & $245(71.4)$ & $63(18.4)$ & $30(8.7)$ & $5(1.5)$ & $343(34.8)$ \\
\hline
\end{tabular}

MPS I, mucopolysaccharidosis type I.

aPercentages are based on the number of patients in each region enrolled in the Registry who met the criteria for inclusion in this analysis as of 2 August 2013.

bPercentages are based on the number of patients in all regions enrolled in the Registry who met the criteria for inclusion in this analysis as of 2 August 2013. 
a
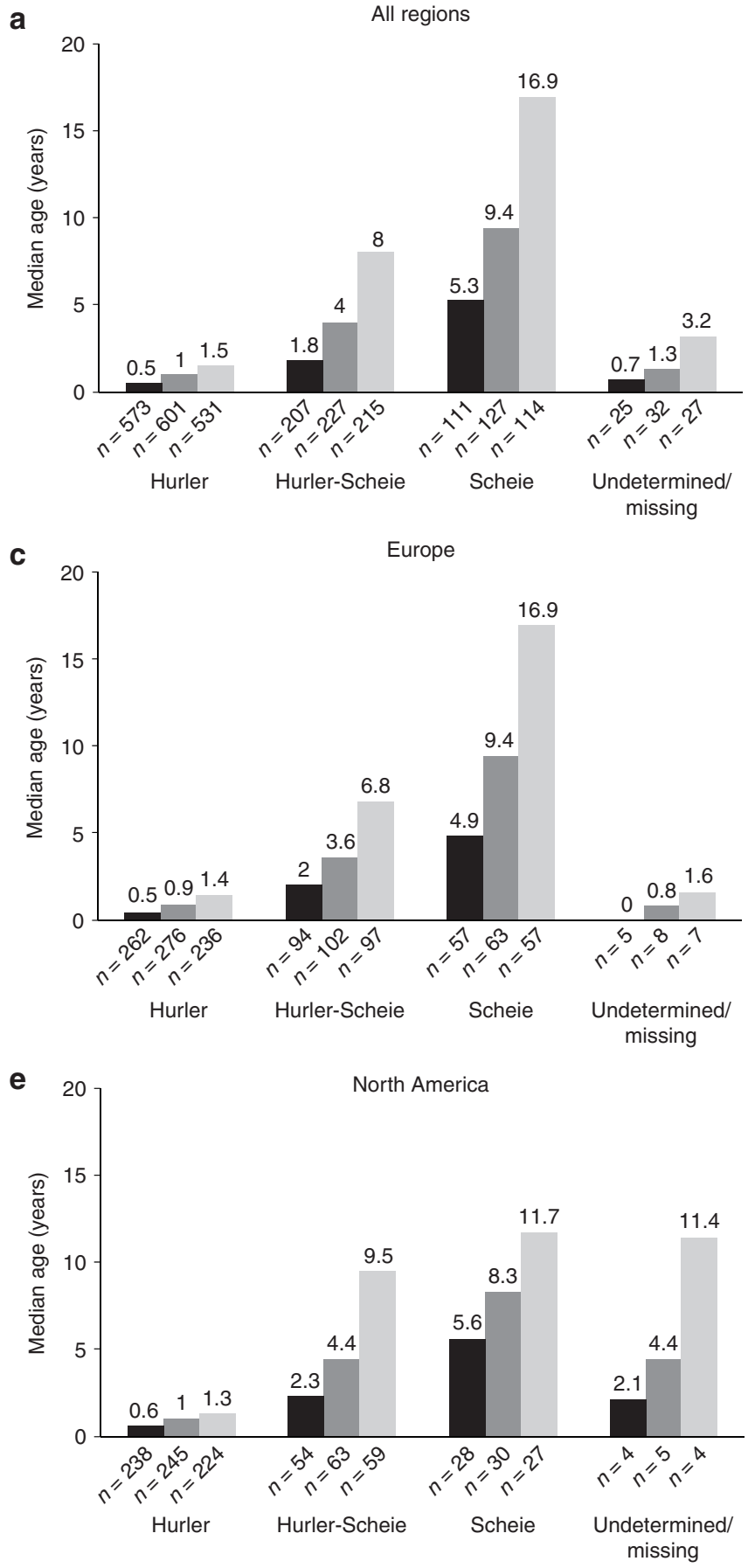
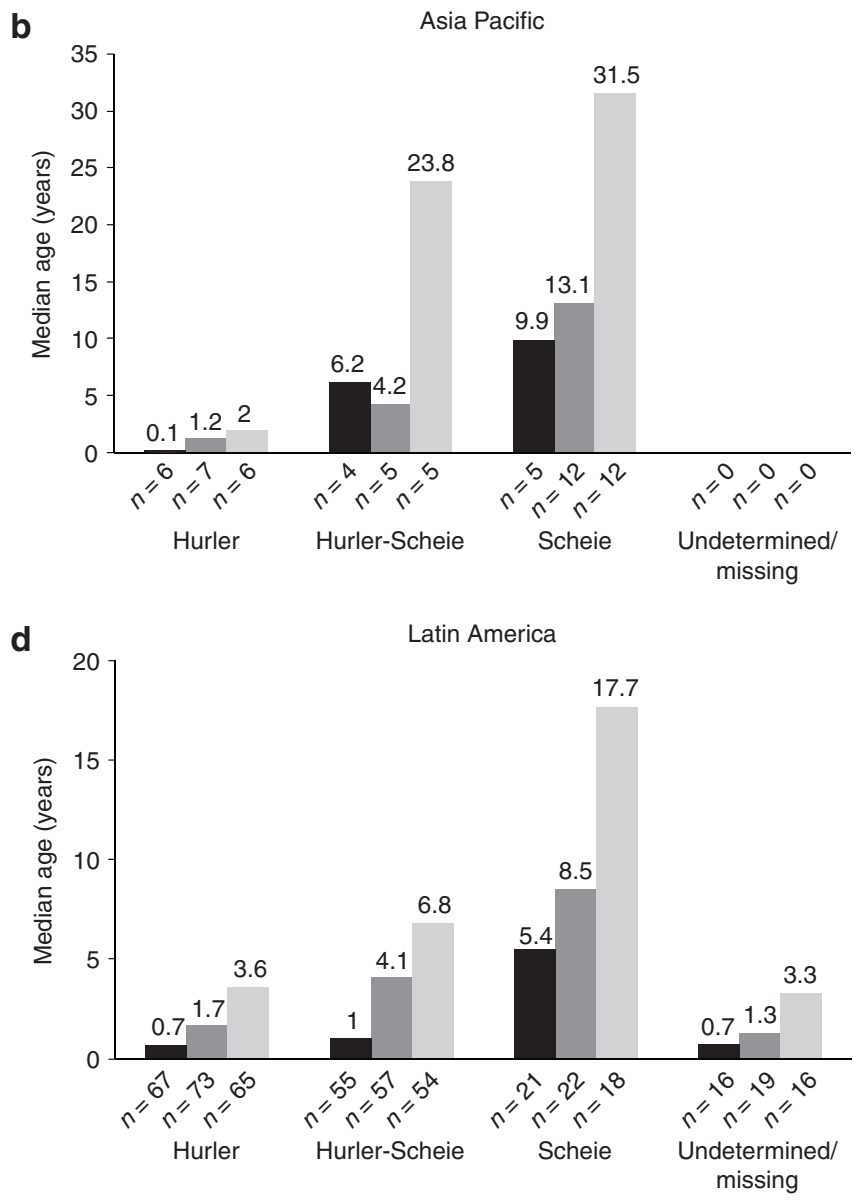

- Symptom onset

- Diagnosis

Treatment

Figure 1 Median age of onset of symptoms, diagnosis, and treatment by phenotype. (a) All regions, (b) Asia Pacific, (c) Europe, (d) Latin America, and (e) North America. Median age in years (left axis) calculated on the basis of patients within each phenotype for whom age of symptom onset, diagnosis, or treatment was recorded. The median age in years is noted above each column. The number of patients, $n$, is noted below each column. In $\mathbf{b}$, the median age of symptom onset is higher than the median age of diagnosis for the five Hurler-Scheie patients, even though all five had an age of symptom onset less than or equal to the age at diagnosis, because data for age of symptom onset were not available for one patient.

Hurler patients. Airway-related symptoms, such as sleep disturbances/snoring were observed in $51.6 \%$ of Hurler patients, $48.9 \%$ of Hurler-Scheie patients, and $26.8 \%$ of Scheie patients. Cognitive impairment was observed in $46.4,31.3$, and $9.4 \%$ of patients with Hurler, Hurler-Scheie, and Scheie phenotypes, respectively.

\section{Age of symptom onset by phenotype}

As expected, first symptoms appeared earlier (within the first 2 years of life) in patients with the Hurler phenotype; whereas, in patients with the Hurler-Scheie and Scheie phenotypes, commonly occurring symptoms were first observed between 3 and 7 , and 5 and 13 years of age, respectively. The median ages of 

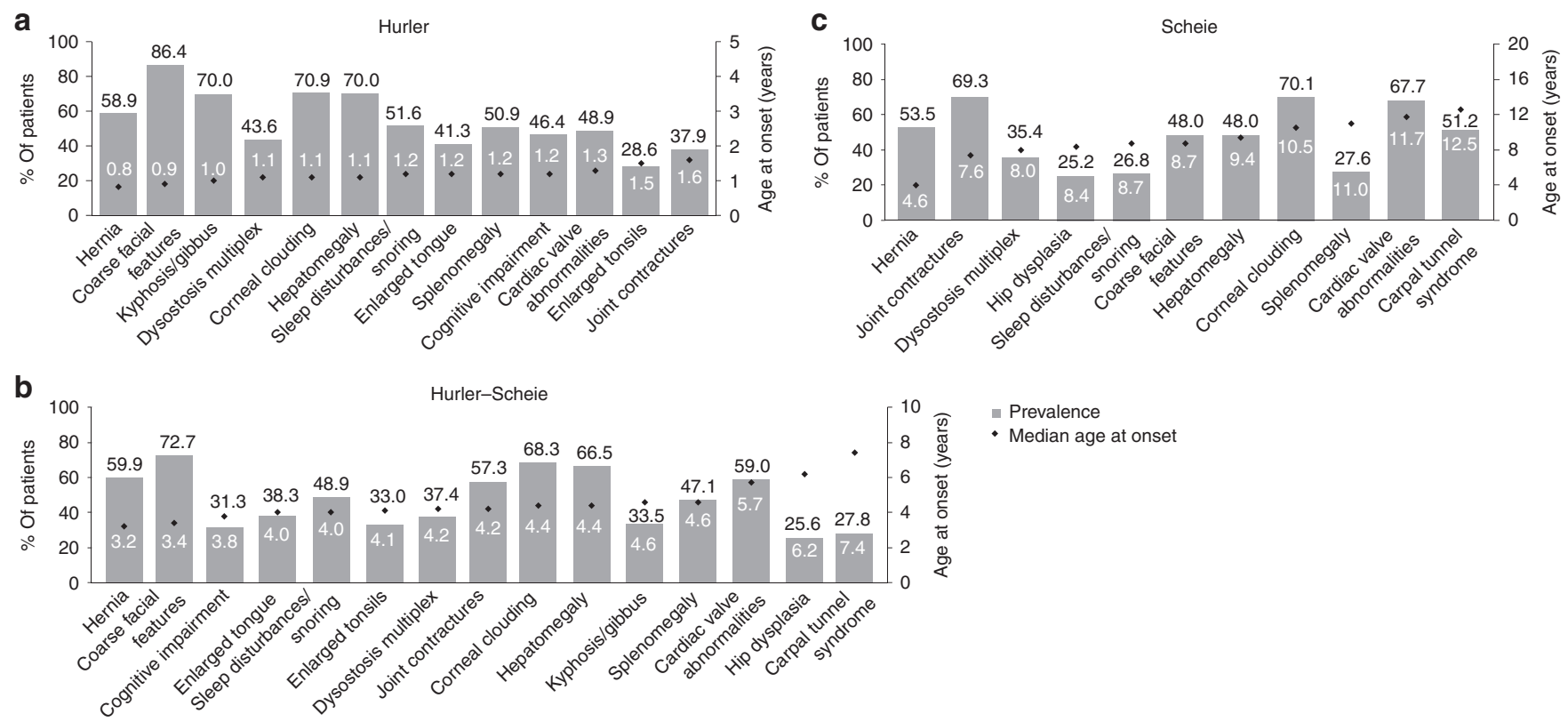

Figure 2 Prevalence and age of onset of signs and symptoms in patients with MPS I by phenotype. (a) Hurler, (b) Hurler-Scheie, and (c) Scheie. Percentages of symptom frequency are shown on the left axis. Only symptoms reported during the natural history period in at least $25 \%$ of patients with the relevant phenotype are shown. Age data are median ages in years (right axis) for those patients with the date of symptom onset recorded. MPS I, mucopolysaccharidosis type I.

onset of symptoms occurring in at least $25 \%$ of patients are presented by phenotype in Figure 2, and the median ages of onset of all symptoms, including those occurring in less than $25 \%$ of patients, are summarized by phenotype in Supplementary Table S2 online.

Across all phenotypes, hernias were the earliest reported symptom, which was noted at median ages of $0.8,3.2$, and 4.6 years of age in Hurler, Hurler-Scheie, and Scheie patients, respectively. Coarse facial features, the most prevalent symptom in patients with Hurler and Hurler-Scheie phenotypes, were also an early finding, with a median age at onset of 0.9 years in Hurler patients and 3.4 and 8.7 years in patients with Hurler-Scheie and Scheie phenotypes, respectively. Corneal clouding, the most prevalent symptom in patients with the Scheie phenotype, was first noted at a median age of 10.5 years in that subset of patients.

Among patients with the Hurler phenotype, kyphosis/gibbus had a median age at onset of 1.0 year (Figure 2a), and dysostosis multiplex, corneal clouding, and hepatomegaly had a median age at onset of 1.1 years. Several symptoms, including sleep disturbances/snoring, enlarged tongue, cognitive impairment, and splenomegaly had a median age at onset of 1.2 years, whereas cardiac valve abnormalities were noted at a median age of 1.3 years. The symptoms that appeared latest in at least $25 \%$ of patients with Hurler syndrome were enlarged tonsils and joint contractures, with a median age at onset of 1.5 and 1.6 years, respectively.

The symptoms reported in Hurler-Scheie patients are shown in Figure 2b. After hernias and coarse facial features, cognitive impairment was the earliest symptom observed, first occurring at a median age of 3.8 years. The median ages of onset for enlarged tongue, sleep disturbances/snoring, enlarged tonsils, joint contractures, and dysostosis multiplex were between 4.0 and 4.2 years. Both corneal clouding and hepatomegaly had a median age at onset of 4.4 years, whereas splenomegaly and kyphosis/gibbus had a median age at onset of 4.6 years. Symptoms appearing later in patients with the Hurler-Scheie phenotype included cardiac valve abnormalities, hip dysplasia, and carpal tunnel syndrome, with median ages at onset of 5.7, 6.2 , and 7.4 years, respectively.

Symptoms reported in at least $25 \%$ of patients with Scheie phenotype are shown in Figure 2c. After hernias, the earliest symptoms within this phenotype were joint contractures and dysostosis multiplex, with median ages at onset of 7.6 and 8.0 years, respectively. Hip dysplasia was first reported at a median age of 8.4 years. Both sleep disturbances/snoring and coarse facial features had a median age at onset of 8.7 years, and hepatomegaly was observed at a median age of 9.4 years. Symptoms appearing later included corneal clouding at 10.5 years, splenomegaly at 11.0 years, cardiac valve abnormalities at 11.7 years, and carpal tunnel syndrome at 12.5 years.

\section{DISCUSSION}

MPS I is a rare panethnic genetic disorder characterized by a spectrum of disease with variable age of onset, progression, and organ involvement. If left untreated, patients with the most severe phenotype experience progressive deterioration of the musculoskeletal, cardiorespiratory, and central nervous systems and, typically, die before the age of 10 years. ${ }^{1}$ Although patients with the least severe phenotype usually have normal 
cognitive functioning and survive into adulthood, more than $50 \%$ may be affected by cardiac valve abnormalities, joint contractures, corneal clouding, hernias, and hepatomegaly. ${ }^{15}$ The availability of improved disease-specific treatments, including HSCT and ERT, allows patients affected with MPS I to obtain substantial clinical benefit for many disease manifestations, including hepatosplenomegaly, upper airway obstruction (including sleep apnea), cardiac symptoms, and coarse facial features. ${ }^{3}$ Importantly, the chances of success from these treatments is improved when initiated prior to the onset of irreversible organ damage.

Due to the rarity of the disease as well as the variability of clinical manifestations, MPS I poses challenges for diagnosis. The MPS I Registry provides the largest global data set for evaluating the natural history, clinical presentation, and potential treatment response of patients with MPS I.

The phenotypic distribution in the Registry data set, with most patients (60.9\%) in the most severe phenotypic category, Hurler, and the remaining more attenuated phenotypes, Hurler-Scheie (23\%) and Scheie (12.9\%), is consistent with existing data, suggesting that $\sim 50-80 \%$ of patients have severe MPS I. ${ }^{4}$ However, the observed rates of the various phenotypes within the Registry may have been impacted by selection bias; e.g., more attenuated phenotypes could be underrepresented due to preferential identification and enrollment of patients with more severe clinical presentations. In an effort to minimize selection bias, the Registry accepts all patients with a diagnosis of MPS I, regardless of treatment status. ${ }^{12}$

Regional differences in phenotypic distribution were also noted. North America and Europe had higher proportions of patients with the Hurler phenotype than did Latin America or the Asia Pacific region. These regional differences could be due to differences in the "genetic landscape" in different geographic regions or may be the result of regional differences in identification and enrollment of MPS I patients in this voluntary Registry. Some evidence exists for regional genetic differences, in that certain severe mutations (common nonsense mutations) identified in North America and Europe have not been observed in the Asia Pacific region. ${ }^{2}$ The profile of MPS I mutations in Brazil also differs from that found in other regions. ${ }^{16}$ Based on the similarities in median ages of symptom onset, diagnosis, and treatment initiation, the present study suggests that a substantial proportion of patients in Latin America whose phenotypes were classified as undetermined/missing could have Hurler syndrome.

Early symptom recognition and diagnosis are essential to achieve the best long-term prognosis in patients with MPS I. It is of paramount importance that not only pediatricians, but other specialists as well, are familiar with the clinical manifestations and consider an MPS diagnosis, especially when symptoms are present in combination with each other. Hernias were the earliest presenting symptoms in all three phenotypes. Therefore, the presence of inguinal or umbilical hernias in young children should raise suspicion of MPS I. Likewise, coarse facial features, which are early manifestations and the most prevalent symptom in patients with Hurler and Hurler-Scheie phenotypes, should be considered as an early sign of a potential MPS I diagnosis. Corneal clouding is also a highly prevalent and relatively early manifestation of MPS I across the phenotypes.

Our findings indicate significant delays in diagnosis and treatment, particularly in patients with attenuated phenotypes. Regional differences were evident as well, with attenuated patients in Asia Pacific receiving treatment 18-22 years following the onset of symptoms-a 10- to 12-year delay compared with the 6-12 years between onset of symptoms and treatment in the overall Registry population. This delay may be due, in part, to a lack of access to ERT in some countries.

The data presented here confirm findings from a previous analysis of Registry data published in 2007, shortly after the Registry was established. ${ }^{12}$ The current analyses are based on a much larger set of patients (987 vs. 302), provide global and regional breakdowns of the data, and include data from the natural history period only. Regardless, and not surprisingly, there are similarities between the two reports in the age at symptom onset, age at diagnosis, and the rates of prevalence of common MPS I symptoms. However, the current report, based on data from a larger number of patients, provides a more detailed and refined analysis of the earlier findings.

When interpreting results of data analyses from the MPS I Registry, certain limitations common to observational registries should be considered. ${ }^{17,18}$ As with any voluntary registry, incomplete or missing data may affect the results, though a recent analysis of the MPS I Registry using source document verification revealed an overall source-to-database error rate of $<4 \%$, with no systematic errors. ${ }^{19}$ It is also possible that assessment and data collection methods at the participating sites around the world may not be sufficiently standardized and/or that Registry enrollment biases, perhaps based on regional disease classification, symptom severity, and symptom reporting practices, affected the results. For example, patients who did not have a particular symptom may not have had any information reported for that symptom (i.e., rather than indicating that a patient did not have a symptom, the investigator did not provide information about that symptom). This possibility led us to calculate symptom prevalence rates as the number of patients with a particular reported symptom divided by the total number of patients in each region and/or phenotype. As a consequence, the true symptom prevalence rates are at least what we have calculated, and may be higher. In this study, a slightly higher than expected number of patients with attenuated MPS I had cognitive impairment (31.3\% of Hurler-Scheie and $9.4 \%$ of Scheie patients). This likely reflects the fact that MPS I presents as a disease continuum, making phenotypic classification of MPS I somewhat subjective. Thus, it is possible that some Hurler and Hurler-Scheie patients may have been misclassified as Hurler-Scheie and Scheie phenotypes, respectively. Although the aforementioned potential limitations are not insignificant, the data compiled in the MPS I 
Registry are clearly valuable for drawing inferences and generating hypotheses.

\section{Conclusions}

The MPS I Registry is the largest global database of information from MPS I patients and provides a useful tool for expanding knowledge about disease presentation, clinical status, and treatment outcomes. Greater understanding of the symptomatology of the disease can lead to earlier diagnosis and initiation of treatment, which may in turn lead to better patient outcomes. Each of the three MPS I phenotypes, Hurler, Hurler-Scheie, and Scheie, is associated with a characteristic constellation of symptoms and disease course. This analysis of data from almost 1,000 patients facilitates definition of the natural history of MPS I across the phenotypic spectrum, which will hopefully increase awareness of the disease and improve early diagnosis. In addition, results from this investigation will be important in establishing benchmarks for future analyses of treatment interventions.

\section{SUPPLEMENTARY MATERIAL}

Supplementary material is linked to the online version of the paper at http://www.nature.com/gim

\section{ACKNOWLEDGMENTS}

The authors are grateful to Kathleen Melia, PhD, SRSC, LLC, for assistance with medical writing, and to Lisa Underhill, MS, and Iva Ivanovska Holder, PhD, Genzyme Global Medical Affairs, for critical review of the manuscript. This study was funded by Genzyme, a Sanofi company.

\section{DISCLOSURE}

M.B. received unrestricted grants, honoraria, and travel support from Genzyme, Shire, Biomarin Pharmaceutical, and Actelion. P.A. is the Chair of the North American Board of Advisors and a member of the International Board of Advisors for the MPS I Registry. R.G. received travel grants and/or speaker honoraria from Genzyme to participate in scientific meetings and investigator fees for his participation in Genzyme-sponsored clinical trials. J.M. received honoraria, educational, and travel grants from Janssen R\&D, Shire, and GreenCross and has participated in clinical trials with BioMarin Phamaceutical, Genzyme, and Shire. T.O. received speaker honoraria from Genzyme to participate in scientific meetings. J.T. and S.F. are employees of Genzyme, a Sanofi company.

\section{REFERENCES}

1. Neufeld EF, Muenzer J. The mucopolysaccharidoses. In: Scriver C, Beaudet A, Sly W, et al. (eds) The Metabolic and Molecular Bases of Inherited Disease. McGraw Hill: New York, NY, 2001:3421-3452.
2. Terlato NJ, Cox GF. Can mucopolysaccharidosis type I disease severity be predicted based on a patient's genotype? A comprehensive review of the literature. Genet Med 2003;5:286-294.

3. Muenzer J, Wraith JE, Clarke LA; International Consensus Panel on Management and Treatment of Mucopolysaccharidosis I. Mucopolysaccharidosis I: management and treatment guidelines. Pediatrics 2009;123:19-29.

4. Peters C, Balthazor M, Shapiro EG, et al. Outcome of unrelated donor bone marrow transplantation in 40 children with Hurler syndrome. Blood 1996;87:4894-4902.

5. Peters C, Shapiro EG, Anderson J, et al. Hurler syndrome: II. Outcome of HLAgenotypically identical sibling and HLA-haploidentical related donor bone marrow transplantation in fifty-four children. The Storage Disease Collaborative Study Group. Blood 1998;91:2601-2608.

6. Kakkis ED, Muenzer J, Tiller GE, et al. Enzyme-replacement therapy in mucopolysaccharidosis I. N Engl J Med 2001;344:182-188.

7. Wraith JE, Clarke LA, Beck M, et al. Enzyme replacement therapy for mucopolysaccharidosis I: a randomized, double-blinded, placebo-controlled, multinational study of recombinant human alpha-L-iduronidase (laronidase). J Pediatr 2004; 144:581-588.

8. Sifuentes M, Doroshow R, Hoft R, et al. A follow-up study of MPS I patients treated with laronidase enzyme replacement therapy for 6 years. Mol Genet Metab 2007;90:171-180.

9. Wraith JE, Beck M, Lane R, et al. Enzyme replacement therapy in patients who have mucopolysaccharidosis I and are younger than 5 years: results of a multinational study of recombinant human alpha-L-iduronidase (laronidase). Pediatrics 2007;120:e37-e46.

10. Clarke LA, Wraith JE, Beck M, et al. Long-term efficacy and safety of laronidase in the treatment of mucopolysaccharidosis I. Pediatrics 2009;123:229-240.

11. Peters C, Balthazor M, Shapiro EG, et al. Outcome of unrelated donor bone marrow transplantation in 40 children with Hurler syndrome. Blood 1996;87:4894-4902.

12. Pastores GM, Arn P, Beck M, et al. The MPS I registry: design, methodology, and early findings of a global disease registry for monitoring patients with Mucopolysaccharidosis Type I. Mol Genet Metab 2007;91:37-47.

13. D'Aco K, Underhill L, Rangachari $L$, et al. Diagnosis and treatment trends in mucopolysaccharidosis I: findings from the MPS I Registry. Eur J Pediatr 2012;171:911-919.

14. Arn $\mathrm{P}, \mathrm{Wraith} \mathrm{JE}$, Underhill L. Characterization of surgical procedures in patients with mucopolysaccharidosis type I: findings from the MPS I Registry. J Pediatr 2009;154:859-64.e3.

15. Thomas JA, Beck M, Clarke JT, Cox GF. Childhood onset of Scheie syndrome, the attenuated form of mucopolysaccharidosis I. J Inherit Metab Dis 2010;33:421-427.

16. Matte U, Leistner S, Lima L, Schwartz I, Giugliani R. Unique frequency of known mutations in Brazilian MPS I patients. Am J Med Genet 2000;90:108-109.

17. Temple R. Problems in the use of large data sets to assess effectiveness. Int $J$ Technol Assess Health Care 1990;6:211-219.

18. Canto JG, Kiefe Cl, Williams OD, Barron HV, Rogers WJ. Comparison of outcomes research with clinical trials using preexisting data. Am J Cardiol 1999;84:923-7, A6.

19. Verhulst K, Artiles-Carloni L, Beck M, et al. Source document verification in the Mucopolysaccharidosis Type I Registry. Pharmacoepidemiol Drug Saf 2012;21:749-752.
This work is licensed under a Creative Commons Attribution-NonCommercial-No Derivative Works 3.0 License. To view a copy of this license, visit http://creativecommons.org/licenses/by-nc-nd/3.0/ 